\title{
Application of a Wet Resonance Grille in a Ventilating Shaft and a Model for Calculating the Efficiency of Dust Removal
}

\author{
Hai-qiao Wang', 2 , Fang-xing Chen', Shi-qiang Chen ${ }^{2,3}$ \\ ${ }^{1}$ School of Resource, Environment and Safety Engineering \\ Hunan University of Science and Technology, Xiangtan, China \\ ${ }^{2}$ Hunan Provincial Engineering Techniques Research Centre of Mine Ventilation \& Dedusting Equipment \\ Hunan University of Science and Technology, Xiangtan, China \\ 1010036@hnust.edu.cn \\ ${ }^{3}$ Work Safety Key Lab on Prevention and Control of Gas and Roof Disasters for Southern Coal Mines \\ Hunan University of Science and Technology, Xiangtan, China \\ shiqiangchen@hnust.edu.cn
}

\begin{abstract}
The dust-removal processes of a spray, wet resonance grille, and water film are considered in an investigation of factors that affect the efficiency of wet resonance-grille dust-removal technology. The dust removal efficiency of the liquid droplet group is deduced adopting limit trajectory theory. A formula for calculating the efficiency of dust removal by a single resonance grille and water film is obtained employing resonance grille filtering and liquid saturation theory. On the basis of Strauss tandem theory, a mathematical model of the total dust removal efficiency is proposed and numerically calculated. Numerical results show that the grille distance-diameter ratio ranges from 2.0 to 2.5 when the liquid-gas ratio is $0.02631 / \mathrm{m}^{3}$ under the condition of low filtration velocity. As the distance-diameter ratio decreases, the filling ratio increases, the grille diameter decreases, and the overall dust removal efficiency increases. The method of calculating the efficiency proposed in this paper can guide engineering practice.
\end{abstract}

Keywords: Wet resonance grille, Total dust removal, Efficiency calculation, Structural parameters, Working parameters.

\section{Introduction}

Blasting is the most commonly used method of mining metal ore. In single blasting, the large explosive dosage produces a high concentration of smoke and leads to flying dust pollution. During the shoveling process, the induced and impacted air flow is strong and dust is again produced. Under the action of wind turbulence, smoke and unsettled dust are discharged into the atmosphere with the return air flow as a form of environmental pollution. It is therefore necessary to purify the exhaust air exiting the ventilating shaft.

From the viewpoint of mine air flow purification, the Peabody Coal Mine first adopted wet-layer dust removal technology in the $1970 \mathrm{~s}{ }^{[1]}$. In the same period, the wet dust collector and wet-layer dust remover were developed by the Maanshan Institute of Mining Research in China; the main functional section is made of wire or nylon mesh ${ }^{[2]}$. Northeast University developed a new type of wet fiber grille dust removal technology in 1998; for a velocity of dusty airflow of 4 $\mathrm{m} / \mathrm{s}$, tests revealed (1) an overall purification efficiency of dust of $99.5 \%$, (2) an arithmetically averaged purification efficiency of $96 \%$ for respiratory dust, and (3) working resistance of $450 \mathrm{~Pa}{ }^{[3]}$. However, the high working resistance is not conducive to the safe and efficient operation of the main fan. Zhang Yinghua applied a wet fiber grille for dust removal at the site of the return airway in 2008; the dust removal efficiency exceeded $95 \%$ when the spray pressure was $4 \mathrm{MPa}$, the diameter of a fiber was $0.250 \mathrm{~mm}$, the spacing between fibers was $0.375 \mathrm{~mm}$, there was a six-layer resonance grating, and the distance between layers was $6.000 \mathrm{~mm}{ }^{[4]}$. However, the power consumption of the pump is huge for high spray pressure.

The Fankou Lead-Zinc Mine in Shaoguan, Guangdong Province, is an underground mine. It adopts blasting to drop ore and mechanical shoveling and uses $4 \mathrm{t}$ of explosive per day. The exhaust flow of the ventilating shaft contains much smoke and dust, which causes serious air pollution. To purify the exhaust air flow of the ventilating shaft, the author puts forward a wet resonance-grille dust-removal system for the ventilating shaft. Furthermore, through theoretical analysis, numerical calculation, and experimental research, the author obtains optimized structural parameters of the resonance grille 
and working parameters of the dust removal system. Field application shows that the system performs well in purifying air and greatly reduces the emission of atmospheric pollutants.

\section{Dust Removal Mechanism of the Wet Resonance-grille Device}

The removal of dust by a wet resonance-grille device mainly involves three parts, namely a spray section, resonancegrille dust-removal section, and dehydration section, as shown in Fig. 1. Among them, the resonance grille is the basis of the device. The resonance grille comprises chord grating wires, a gap between wires, and a maintenance structure, as shown in Fig. 2. The maintenance structure is a rigid frame of a certain size. In this maintenance structure, stainless-steel wire having a tiny diameter is spun to form chord grating wires, which are separated by a certain gap. The spray droplets and airflow affect the chord wire and force its elastic disturbance to realize the vibration of the chord wire. The wet resonance grille comprises resonance chord grating wire and water films attached to the wire and each other.

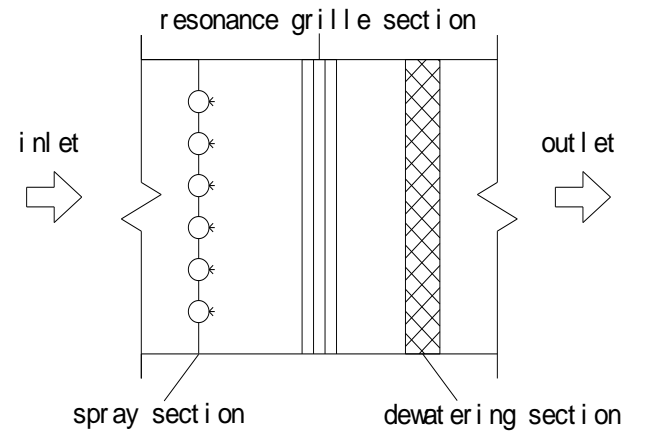

Fig. 1: Dust removal of a wet resonance grille.

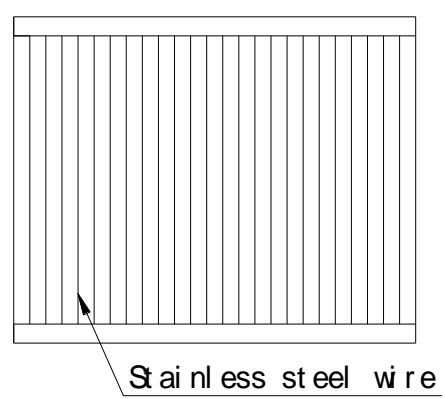

Fig. 2: Section of the resonance grill.

Wet resonance-grating dust removal combines a water droplet, resonance grille, and water film. The droplet group in the spray section not only results in the inertial collision, interception, and diffusion of dust but also forms a film of water between chord grating wires. The resonance grille intercepts and collides with dust while the water film also intercepts dust, thus improving the dust removal effect. The process of dusty airflow passing through the water film is similar to forced scrubbing of this airflow. When the water film is damaged, the film breaks into droplets; these droplets then come together and coagulate into water flow, which washes down the dust particles. Water mist is removed by a water-retaining plate. Clean exhaust air finally flows into the atmosphere.

When the ratio of wire spacing $T$ to wire diameter $\mathrm{d}_{\mathrm{f}}$ is between 2.0 and 2.5, the flow around the static resonant grille forms a cylindrical wake flow of the chord grating wire, and the wake flow then forms a Karman vortex street ${ }^{[5]}$. The vortex street frequency is the number of times a vortex sheds off a cylinder in 1 second. The chord grating wire resonates when the vortex street frequency is the same as the natural frequency of the chord grating wire. When resonance occurs, the spacing between wires will be larger, equal to, or smaller than the original gap. When the gap is reduced to a certain extent, the droplets attached to the wire form a water film in the gap under the action of the dual chord grating wire. With the continuation of vibration, the stretching of the wire works to overcome the surface tension of the droplets, and the area of the water film continues to increase. When the area of the water film increases to a certain extent, airflow affects the water film and the water film then breaks down, and a fine water body condenses on the chord grid wire. In this way, a life cycle of the formation, expansion, and rupture of the water film is completed. The life cycle of the water film is affected by many factors, such as the velocity of the dusty airflow, the mechanical properties of the chord grating wire, the structural parameters of the resonance grating, the properties of the solids, liquid, and gas, and the degree of saturation ${ }^{[6]}$.

In terms of the effect of dust removal, the total dust removal efficiency of wet resonant grids is mainly affected by the pre-collection efficiency of dust particles by droplets, the dust removal efficiency of the chord grating, and the dust removal efficiency of the water film in the gap.

\section{Calculation Model of the Dust Removal Efficiency}

Authors are asked to replace the "XXX" number (with the paper code that was assigned when the paper was accepted) on the header of the first page and on the footer of other pages in order to set a unique page number in the Proceedings. 


\subsection{Collection efficiency of the water droplet}

The droplets is spurted from the spray section pre-collect the dust in the airflow, and the efficiency of this collecting is thus important. Limit trajectory theory can be applied in calculating the efficiency. When the pre-collection effect of a droplet is considered separately, the dust collecting efficiency is calculated as ${ }^{[7,8]}$

$$
\eta_{1}=1-\exp \left(-\frac{2 \eta_{\mathrm{PR}} \rho_{\mathrm{c}} D_{\mathrm{c}} Q_{\mathrm{c}} u_{\mathrm{r}}}{55 Q_{0} \mu}\right)
$$

where $\eta_{1}$ is the pre-collection efficiency of droplets for dust; $\eta_{\mathrm{PR}}$ is the total dust collection efficiency of a single droplet, dimensionless; $Q_{\mathrm{c}}$ and $Q_{0}$ are respectively the droplet and dusty air flow rates, $\mathrm{m}^{3} / \mathrm{s} ; \rho_{\mathrm{c}}$ is the droplet density, 1000 $\mathrm{kg} / \mathrm{m}^{3} ; u_{\mathrm{r}}$ is the relative velocity of the undisturbed dusty air and droplets in the upstream, with the velocity of dusty air being $2-6 \mathrm{~m} / \mathrm{s} ; D_{\mathrm{c}}$ is the qualitative size of droplets, $\mu \mathrm{m}$; and $\mu$ is the aerodynamic viscosity, $1.8 \times 10^{-5} \mathrm{~Pa} \cdot \mathrm{s}$.

In formula (1), the ratio of the droplet airflow to dusty airflow is the liquid-gas ratio. The liquid-gas ratio directly affects the pre-collection efficiency of the droplet in formula (1) and the total efficiency of the dust removal system. Gong Lihong carried out an experimental study on wet vibration fiber grille dust removal and found that the dust removal efficiency of the whole system increased from $76.9 \%$ to $90.2 \%$ when the liquid-gas ratio increased from 0.0175 to 0.0292 $1 / \mathrm{m}^{3}$ but no longer increased appreciably as the liquid-gas ratio increased further ${ }^{[9]}$. Additionally, Liu Jun and others found that wind speed had little effect on the dust removal efficiency ${ }^{[10]}$. Yao Xiaoqing put forward the wet dense fiber grille, which has good efficiency but large resistance ${ }^{[11]}$. Zhao Jie measured the flow resistance downstream and upstream of the metal fiber grille and found that the deviation between the theoretical calculation and measurement was $20 \%$ at high wind speed ${ }^{[12,13]}$.

Supposing the average droplet diameter of water mist is $70 \mu \mathrm{m}$ (according to technical parameters of the Kirinoikeuchi small-flow nozzle), a liquid-gas ratio of $0.0263 \mathrm{l} / \mathrm{m}^{3}$, an exhaust wind speed range of $4 \mathrm{~m} / \mathrm{s}$, and a total dust collection efficiency of a single droplet of $38 \%{ }^{[9]}$, the efficiency of the pre-collection of dust by water mist in formula (1) remains at about $15 \%$.

\subsection{Dust collection efficiency of the resonance grille}

Figure 3 shows the purification efficiencies of the main interception, inertial collision, and diffusion effect in the absence of spraying ${ }^{[14]}$. The figure shows that the dominant effect is inertia and interception when the particle size is larger than $1 \mu \mathrm{m}$.

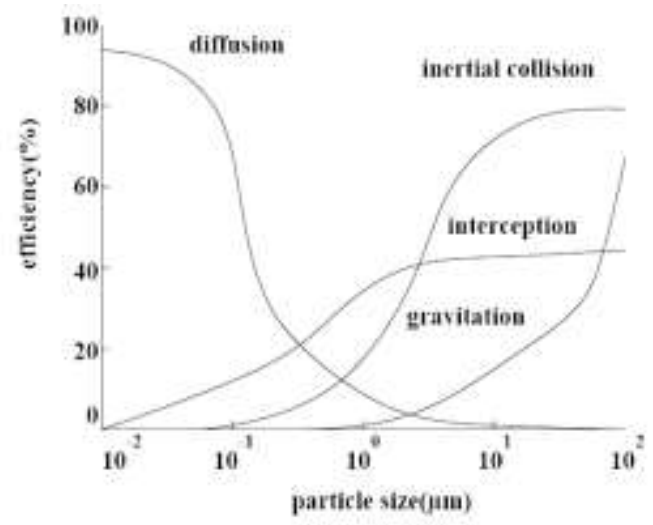

Fig. 3: Purification efficiencies of different mechanisms.

Analysis of the dust collection efficiency of the resonance grille involves determining the flow pattern of dust-laden air flow around a chord grating wire. The Reynolds number is generally used as the basis for determining the flow pattern. According to the expressions of the inertial force and viscous force, the Reynolds number (Re) can be expressed as

$$
\operatorname{Re}=\rho u_{0} d_{\mathrm{f}} / \mu
$$


where $\rho$ is the density of airflow, $\mathrm{kg} / \mathrm{m}^{3} ; d_{\mathrm{f}}$ is the diameter of the chord grating wire, $\mathrm{mm}$; and $u_{0}$ is the upstream undisturbed air velocity, taking values in the range of $2-6 \mathrm{~m} / \mathrm{s}$.

Formula (2) is used to calculate Re. According to inviscid potential flow theory, when Re is between 60 and 120, the flow can be approximately treated as potential flow. In the application of the potential-flow method, the collection efficiency of the inertial collision of the dust with the chord grating wire is ${ }^{[15]}$

$$
\eta_{\mathrm{st}}=\left(\frac{S_{\mathrm{t}}}{S_{\mathrm{t}}+0.35}\right)^{2}
$$

where $\eta_{\mathrm{st}}$ is the efficiency of the inertial collision of the chord grating wire with dust, dimensionless, and $S_{\mathrm{t}}$ is the inertial collision parameter, dimensionless.

In formula (3), the inertial collision parameters are calculated as

$$
S_{\mathrm{t}}=\frac{C d_{\mathrm{p}}^{2} \rho_{\mathrm{p}} u_{0}}{18 \mu d_{\mathrm{f}}}
$$

where $C=1+0.165 / d_{\mathrm{p}}$ is a correction factor, dimensionless; $D_{\mathrm{p}}$ is the particle size of dust, $\mu \mathrm{m}$; and $\rho_{\mathrm{p}}$ is the dust density, taking a value of $2000 \mathrm{~kg} / \mathrm{m}^{3}$ (according to the density of coal dust).

In the case of formula (4), the chord grating wire as a trap for dust interception is under the action of a spray while the chord grating wire is wrapped by water to form a wet chord grating wire. When there is dusty airflow around the wet chord grating wire, once the distance between the streamline and the surface of the wire is within the range of $d_{p} / 2$, the dust is considered to be in contact with and thus collected by the wet wire.

The main factor affecting the interception is the size of dust particles. $R=d_{\mathrm{p}} / d_{\mathrm{f}}$ is used as the interception parameter. The interception efficiency is ${ }^{[15]}$

$$
\eta_{\mathrm{DI}}=\frac{R(2+R)}{1+R}
$$

where $\eta_{\mathrm{DI}}$ is the dust interception efficiency of the chord grating wire, dimensionless, and $R$ is the interception parameter, dimensionless.

In fact, the interception effect and inertial collision collecting effect exist simultaneously. According to Strauss series theory, the comprehensive efficiency can be calculated as a series of dust removal ${ }^{[7]}$ :

$$
\eta_{\mathrm{f}}=1-\left(1-\eta_{\mathrm{st}}\right)\left(1-\eta_{\mathrm{DI}}\right)
$$

where $\eta_{\mathrm{f}}$ is the comprehensive efficiency of the chord grating wire collecting dust.

In the absence of spray, the catching of dust by the dense dry chord grating wire is similar to filtration by a fiber layer. The theoretical formula for calculating the dust removal efficiency of dense dry chord grating wire is ${ }^{[14,16]}$

$$
\eta_{\mathrm{f} 1}=1-\exp \left(-\frac{4 \beta \eta_{\mathrm{f}}}{\pi(1-\beta)}\right)
$$

where $\eta_{\mathrm{fl}}$ is the dust collection efficiency of the dense dry chord grating wire, dimensionless, and $\beta$ is the filling coefficient of the dense chord grating wire, dimensionless.

\subsection{Dust Collection Efficiency of the Water Film in the Gap Between Chord Grating Wires}

The collection of dust by the water film in the gap between chord grating wires is the basis of dust removal by a wet resonance grille. A water film forms in the gap between wires when the chord grating wire resonates. The following 
assumptions are made for the water film. The thickness of the water film is the same as the diameter of the chord wire. The droplet and air in the main phase that forms the water film are evenly dispersed in the flow basin that forms in the interstitial gap between wires. In the flow zone, the maximum percentage of the fluid volume occupied by water film is the ideal saturation. Under a strong capillary force, the fraction of the volume occupied by the water film, which cannot be driven and displaced by the dusty airflow, is the bound saturation. The volume fraction that can be displaced is the effective saturation. The relationship among the three saturations is ${ }^{[16]}$

$$
S_{\mathrm{e}}=\frac{S_{\mathrm{w}}-S_{\mathrm{w} 0}}{1-S_{\mathrm{w} 0}}
$$

where $S_{\mathrm{e}}$ is the effective saturation, dimensionless; $S_{\mathrm{w}}$ is the ideal saturation determined by experiment, dimensionless; and $S_{\mathrm{w} 0}$ is the bound saturation determined by experiment, dimensionless.

At bound saturation $S_{\mathrm{w} 0}$, the filling coefficient $\beta_{\mathrm{w}}$ of the wet resonance grille is

$$
\beta_{\mathrm{w}}=\beta+(1-\beta) S_{\mathrm{w} 0}
$$

When the chord grating wire resonates, the active surface area of the water film between wires is

$$
A_{\mathrm{w}}=\left(1+f_{\mathrm{f}}\right) \cdot\left(1-\beta_{\mathrm{w}}\right) A S_{\mathrm{e}}
$$

where $A_{\mathrm{w}}$ is the active surface area of the water film, $\mathrm{m}^{2} ; A$ is the resonance grille area, $\mathrm{m}^{2}$; and $f_{\mathrm{f}}$ is the frequency of the water film formation, which is twice the vibration frequency of the chord grating wire ${ }^{[16]}$. The vibration frequency of the chord grating wire is equal to the dimensionless frequency of the vortex shedding. The Strohal number $S_{\mathrm{r}}=0.198(1-$ $19.7 / \mathrm{Re})^{[17]}$.

In the process of the collection of dust by the water film between wires, dust is considered to be collected by the water film between wires if there is contact between the dust and water film. The dust collection efficiency of the water film is

$$
\eta_{\mathrm{w}}=\frac{A_{\mathrm{w}}}{A}=\left(1+f_{\mathrm{f}}\right)\left(1-\beta_{\mathrm{w}}\right) S_{\mathrm{e}}
$$

where $\eta_{\mathrm{w}}$ is the dust collection efficiency of the water film between wires, dimensionless.

\subsection{Comprehensive Dust Collection by the Wet Resonance Grille}

Dust removal by the wet resonance grille comprises the collection of dust by the water film and dry resonance grille. The comprehensive collection of dust by the wet resonance grille is then

$$
\eta_{2}=1-\left(1-\eta_{\mathrm{w}}\right)\left(1-\eta_{\mathrm{f} 1}\right)
$$

where $\eta_{2}$ is the comprehensive dust collection efficiency of the wet resonance grille, dimensionless.

\section{Numerical Calculation of the Dust Removal Efficiency}

In the case of spray, the dust removal process of a wet resonance grille, which comprises a double layer of chord grating wire, can be regarded as the series actions of dust collection by a droplet, dust collection by a dry chord grating wire, and dust collection by the water film between two wires. The (dimensionless) total dust removal efficiency of the dust removal section of a wet resonance grille is

$$
\eta=1-\left(1-\eta_{1}\right)\left(1-\eta_{2}\right)^{2}
$$


The filling coefficient of the resonant grille is $0.314-0.341$ when the distance-diameter ratio is $2.0-2.5$. The bound saturation is supposed as 0.25 when the liquid-gas ratio is $0.0263 \mathrm{~L} / \mathrm{m}^{3}{ }^{[9]}$. Initial data and boundary variables, such as the filtration velocity, chord grating wire diameter, and dust particle size, are input into formulas (1), (12), and (13). The effects of boundary variables on the performance of the wet resonance-grille dust-removal system comprising two layers of chord grating wire are obtained, as shown in Fig. 4.

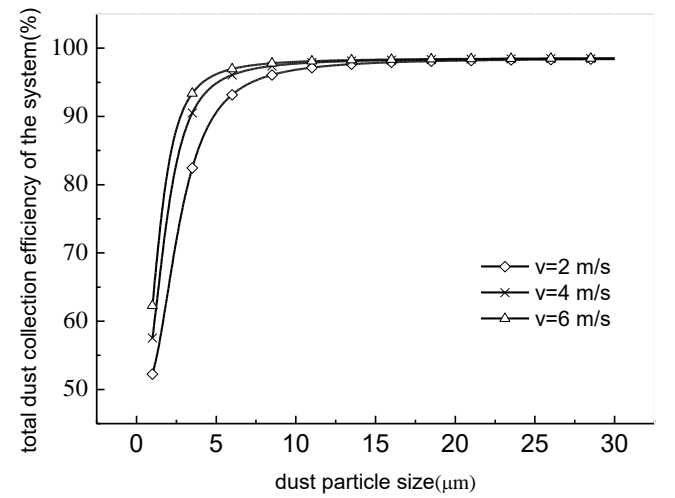

(a) Filtration velocity

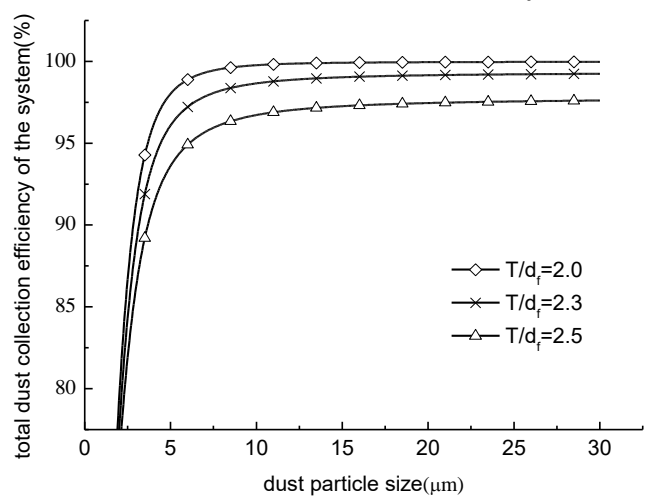

(b) Spacing ratio

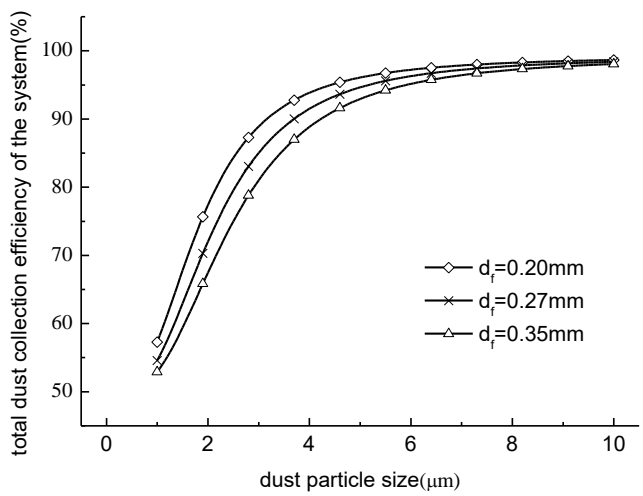

(c) Diameter of the chord grating wire

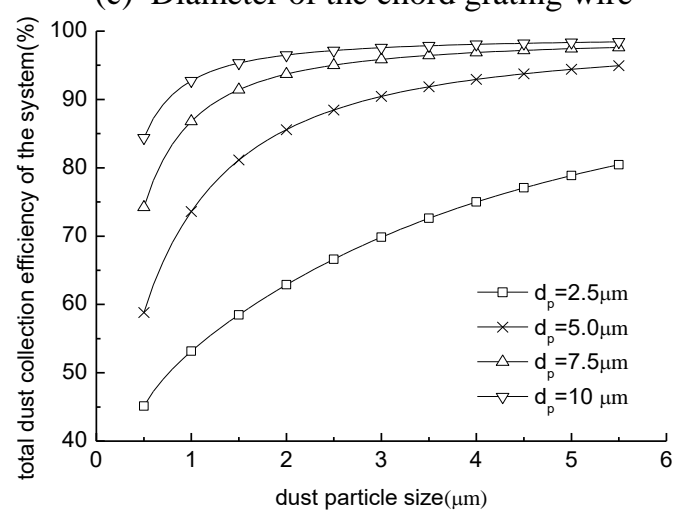

(d) Dust size

Fig. 4: Effects of four variables on the efficiency of dust removal.

Figure 4 (a) shows the total dust removal efficiency of the wet resonance grating system varying with the filtration velocity when the distance-diameter ratio is constant. It is seen that the total dust removal efficiency increases with the filtration velocity. More specifically, with an increase in velocity, the rate of collection of dust below $10 \mu \mathrm{m}$ changes more obviously. The collection efficiency of dust above $10 \mu \mathrm{m}$ is basically the same under each filtration velocity and converges to about $96 \%$.

Figure 4 (b) shows the classification efficiency for different distance-diameter ratios. It is seen that the total dust removal efficiency decreases with an increase in the distance-diameter ratio. This indicates that the probability of collision between dust and the resonance grille is reduced. Obviously, the filling coefficient, directly affecting the collision probability, is inversely correlated with the distance-diameter ratio. Therefore, when the distance-diameter ratio increases, the filling coefficient decreases, the collision probability decreases, and the total dust removal efficiency therefore decreases.

Figure 4 (c) shows the total efficiency of the wet resonance-grille dust-removal system for different diameters of wire. It is seen that the collection rate of respiratory dust within $10 \mu \mathrm{m}$ increases rapidly with the dust particle size when the filling coefficient and distance-diameter ratio remain the same. Moreover, the total dust removal efficiency decreases with an increase in the wire diameter.

Figure 4 (d) compares the classification efficiency for four dust particle sizes; i.e., 2.5, 5.0, 7.5, and 10.0 $\mu \mathrm{m}$. The figure shows the following. (1) The particle size does not affect the trend of the change in the total dust removal efficiency. (2) The efficiency is low at a low filtration velocity. The total dust removal efficiency increases rapidly with an increase in 
the filtration velocity. Moreover, the efficiency increases with the particle size. (3) The change in the total dust removal efficiency is relatively gentle in the higher filtration velocity range. (4) The larger the particle size of the dust, the more stably the total dust removal efficiency changes with an increase in the filtration velocity.

In summary, when the liquid-gas ratio is $0.0263 \mathrm{~L} / \mathrm{m}^{3}$, the range of the distance-diameter ratio is determined to be 2.0-2.5. It is necessary to reduce the diameter of chord grating wire and the distance-diameter ratio to improve the filling coefficient of the resonance grille. The above structural and operational parameters obtained are important references for the design of the wet resonance-grille dust-removal system.

\section{Engineering Application and Effect}

\subsection{Design and Fabrication of the Wet Resonance-grille Dust-removal System for a Ventilating Shaft}

The GAF28-133-1 main fan installed in the Xinnan air shaft at the Fankou Lead-Zinc Mine has air flow of 220-260 $\mathrm{m}^{3} / \mathrm{s}$, full pressure of $1878-2527 \mathrm{~Pa}$, rated speed of $985 \mathrm{r} / \mathrm{min}$, and rated power of $1250 \mathrm{~kW}$.

A wet resonance-grille dust-removal system of the mine duct was designed and installed in the horizontal duct of the outlet section of the main fan. The net section of the duct has dimensions of $8 \mathrm{~m} \times 8 \mathrm{~m}$. There are $100 \mathrm{SS}-10 \mathrm{~W}$ stainlesssteel wide-angle nozzles. Two layers of chord grating wire constitute a single resonance grille plate, which is made of stainless steel and comprises 100 pieces. There are 64 pieces of a W-type stainless-steel water retainer. Additionally, there is an 80GDL-50-20×3 multistage water pump with a flow rate of $50 \mathrm{~m}^{3} / \mathrm{h}$ and a water head of $60 \mathrm{~m}$. The water system has a Y-type filter with a diameter of $0.282 \mathrm{~mm}$. A $240-\mathrm{m}^{3}$ circulating sedimentation pool was constructed to save water. Dust is removed by the wet resonance grille in the duct, as shown in Fig. 5.

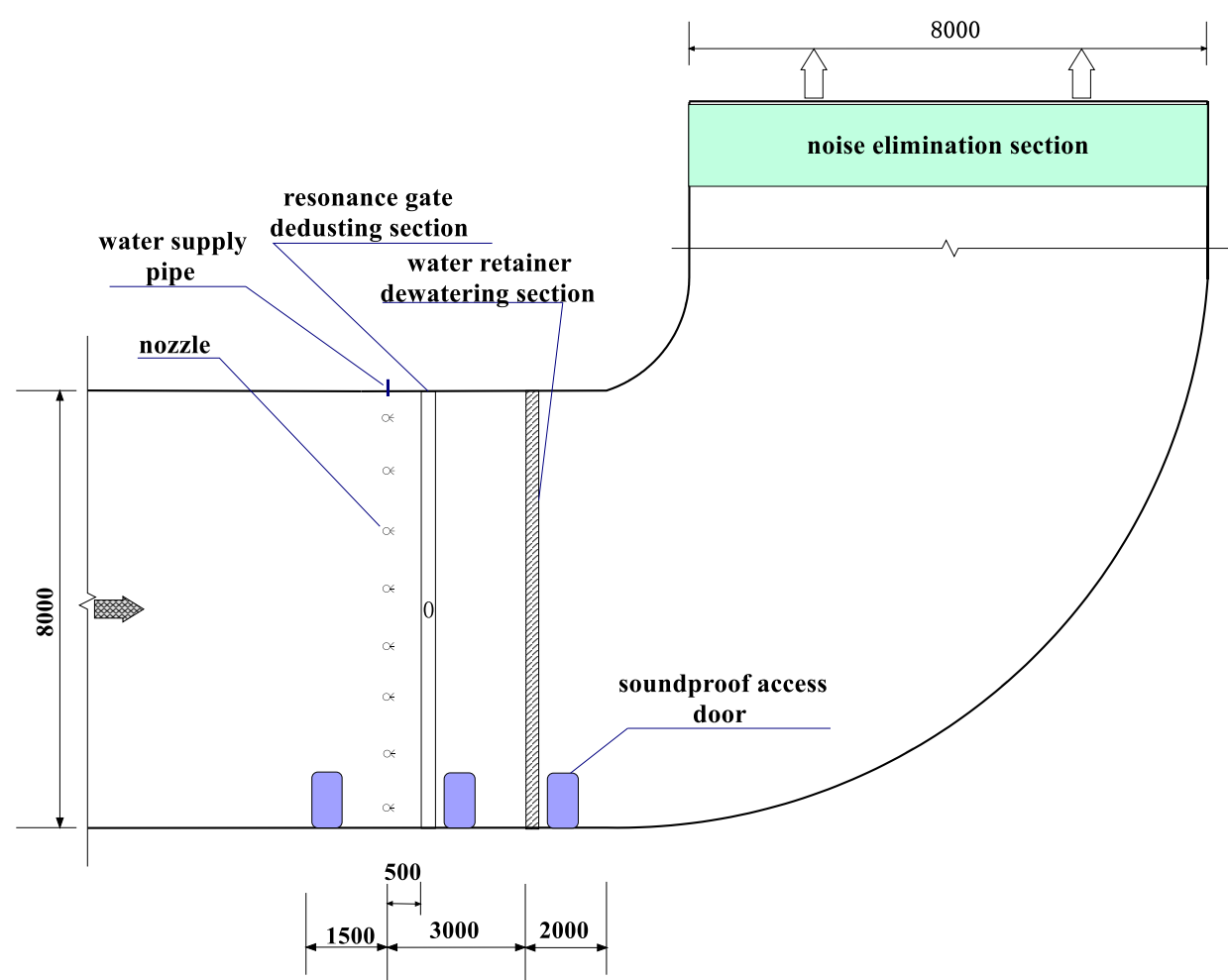

Fig. 5: Wet resonance grille for dust removal in the roadway of an air shaft.

The installed resonance grille and W-type water-retaining plate are respectively shown in Figs. 6 and 7. 


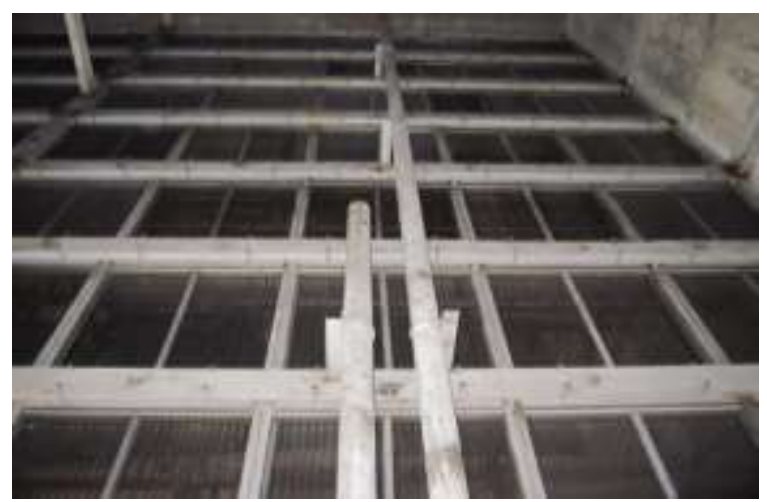

Fig. 6: Resonance-grille group .

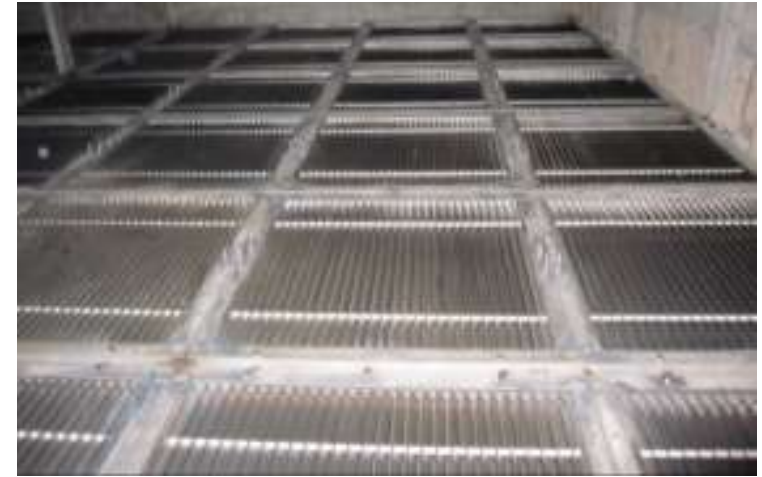

Fig. 7: W-type water-retaining plate group.

\subsection{Measurement of the Dust Removal Efficiency and Benefits}

The overall performance of the dust removal system was tested after installation. The location of the first experimental sectional area is $3 \mathrm{~m}$ before the dust-removal-device section, while the second experimental sectional area is after the dustremoval device section and located in the outlet of the main fan diffuser. Six sampling points were selected for each experimental area according to the method of equal areas. Under normal ventilation, dust was collected by a CCZ-20 dust sampler and then weighed by a Discover DV215CD. Dust concentrations were finally obtained by measuring the dust weights in the first and second areas, and the total dust removal efficiency was calculated as ${ }^{[18]}$

$$
\eta=\frac{c_{1}-c_{2}}{c_{1}} \times 100 \%
$$

where $c_{1}$ and $c_{2}$ are respectively the dust concentrations before and after dust removal, $\mathrm{mg} / \mathrm{m}^{3}$.

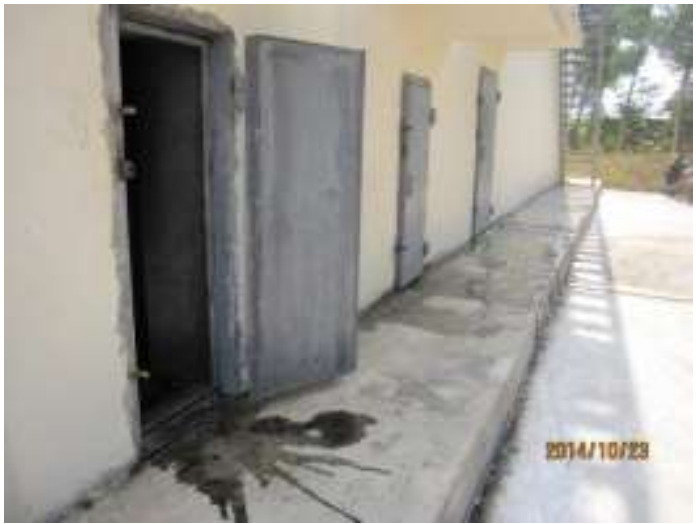

Fig. 8: Sewage water before and after the device.

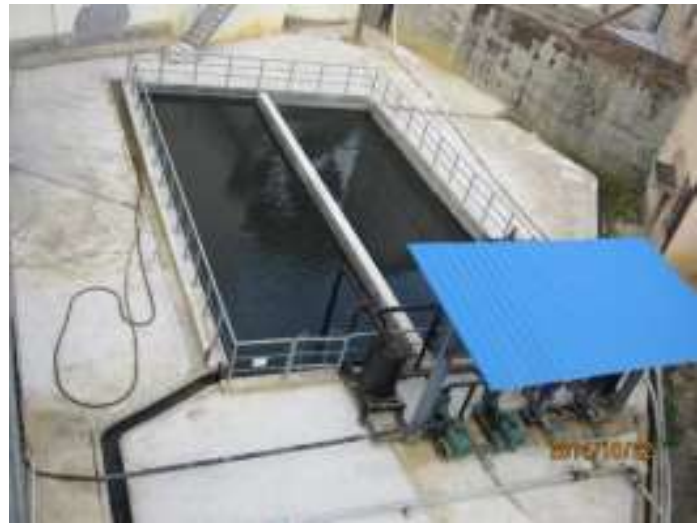

Fig. 9: Recirculation pool after 1 week.

Twelve groups of data were obtained by sampling six times before and after the dust removal device for a sampling time of $10 \mathrm{~min}$ and sampling flow of $20 \mathrm{~L} / \mathrm{min}$. The data are shown in Table 1. 
Table 1: Data recorded at the site and total dust removal efficiency.

\begin{tabular}{|c|c|c|c|c|c|c|c|c|}
\hline $\begin{array}{c}\text { measur } \\
\text { ement }\end{array}$ & $\begin{array}{c}\text { Samplin } \\
\text { g point }\end{array}$ & $\begin{array}{l}\text { Accum } \\
\text { ulated } \\
\text { gas } \\
\text { product } \\
\text { ion } \\
\text { L } \\
\end{array}$ & $\begin{array}{c}\text { Filter } \\
\text { membrane } \\
\text { weight } \\
\text { before } \\
\text { sampling } \\
\text { g } \\
\end{array}$ & $\begin{array}{c}\text { Filter } \\
\text { membrane } \\
\text { weight } \\
\text { after } \\
\text { sampling } \\
\text { g } \\
\end{array}$ & $\begin{array}{l}\text { Dust the } \\
\text { increment } \\
\text { al } \\
\text { g }\end{array}$ & $\begin{array}{c}\text { Dust } \\
\text { concentrati } \\
\text { on } \\
\mathrm{mg} / \mathrm{m}^{3}\end{array}$ & $\begin{array}{c}\text { Average } \\
\text { concentr } \\
\text { ation } \\
\mathrm{mg} / \mathrm{m}^{3}\end{array}$ & $\begin{array}{c}\text { Total } \\
\text { dust } \\
\text { removal } \\
\text { efficienc } \\
\text { y } \\
\% \\
\end{array}$ \\
\hline \multirow{6}{*}{$\begin{array}{l}1 \mathrm{st} \\
\text { area }\end{array}$} & $1-1$ & 200 & 0.06714 & 0.076935 & 0.009795 & 48.975 & \multirow{6}{*}{43.9} & \multirow{12}{*}{90.6} \\
\hline & $1-2$ & 200 & 0.06565 & 0.072795 & 0.007145 & 35.725 & & \\
\hline & $1-3$ & 200 & 0.06722 & 0.076390 & 0.009170 & 45.850 & & \\
\hline & $1-4$ & 200 & 0.06653 & 0.074940 & 0.008410 & 42.050 & & \\
\hline & $1-5$ & 200 & 0.06875 & 0.078270 & 0.009520 & 47.600 & & \\
\hline & $1-6$ & 200 & 0.06582 & 0.074435 & 0.008615 & 43.075 & & \\
\hline \multirow{6}{*}{$\begin{array}{l}2 \text { nd } \\
\text { area }\end{array}$} & $2-1$ & 200 & 0.06908 & 0.069990 & 0.000910 & 4.550 & \multirow{6}{*}{4.1} & \\
\hline & $2-2$ & 200 & 0.06731 & 0.068125 & 0.000815 & 4.075 & & \\
\hline & $2-3$ & 200 & 0.07204 & 0.072865 & 0.000825 & 4.125 & & \\
\hline & $2-4$ & 200 & 0.06778 & 0.068425 & 0.000645 & 3.225 & & \\
\hline & $2-5$ & 200 & 0.06660 & 0.067575 & 0.000975 & 4.875 & & \\
\hline & $2-6$ & 200 & 0.07131 & 0.072095 & 0.000785 & 3.925 & & \\
\hline
\end{tabular}

Field operation of the system showed that (1) the resistance of the dust removal device is $210 \mathrm{~Pa}$, which is significantly lower than that of other conventional dust removers, (2) the total dust removal efficiency is $90.6 \%$ and the exhaust air meets atmospheric emission standards, and (3) the blasting smoke is effectively washed and absorbed. Water leaking from the duct repair door is black before the dust removal device and clear after the dust removal device, as shown in Fig. 8. Additionally, after the water droplets wash the dusty flow, the water in the circulating pool gradually becomes blacker and blacker, and there is sediment in the pool, as shown in Fig. 9. Water in the circulating pool should be replaced once a week to save water.

\section{Conclusion}

(1) The wet resonance-grating dust-removal system has the characteristics of high efficiency, low resistance, and little effect on the ventilation system and it is thus suitable for the purification of exhaust airflow in a shaft.

(2) Theoretical analysis and numerical calculation show that when the liquid-gas ratio is $0.0263 \mathrm{~L} / \mathrm{m}^{3}$ and the distance-diameter ratio is 2.0-2.5, as the distance-diameter ratio decreases, the filling coefficient increases, the diameter of steel wire decreases, and the fractional dust removal efficiency of the wet resonance grille increases.

(3) In field application at the Fankou Lead-Zinc Mine, the average dust removal efficiency of the wet resonance-grille dust-removal system in a duct reaches $90.6 \%$ and the average concentration of dust in the exhaust is $4.1 \mathrm{mg} / \mathrm{m}^{3}$, which marks a large reduction in air pollution from the mine exhaust to the benefit of the environment.

\section{Acknowledgements}

The authors are grateful for financial support from the National Natural Science Foundation of China (Grant Nos. 51774134, 51864014, and 51574123), the Project of Hunan Provincial Natural Science Foundation of China (Grant No. 2019JJ60044), and the Doctor Research Fund of Hunan University of Science and Technology (Grant No. E51681). We 
thank Glenn Pennycook, MSc, from Liwen Bianji, Edanz Group China (www.liwenbianji.cn/ac), for editing the English text of a draft of this manuscript.

\section{References}

[1] G. Knight, "Generation and control of mine airborne dust," in the Proceeding of the 2nd US Mine Ventilation Symposium, Ottawa, 1985.

[2] I. E. Agranovski, J. M. Whitecombe, "Utilisation of wet fibrous media for filtration of sticdy aerosol particles," Journal of Aerosol Science, vol. 31, pp. 204-205, 2000.

[3] Gong Lihong, Wang Yingmin, Liu Tiemin, et al, "Study on Mechanism and Efficiency of Vibration Dust Removal of Wet Fiber Grids," Journal of Safety and Environment, vol. 3, no. 3, pp. 32-35, 2003.

[4] Zhang Yinghua, Huang Zhian, Ge Shiyou, "Study on Dust Removal Efficiency of Wet Fiber Grid in Return Air Lane," in Proceedings of International Symposium on Safety Science and Technology, Shenyang, Northeastern University Press, 2008.

[5] Chen Wenqu, "Study on flow and vortex induced vibration of two-dimensional series parallel columns," Zhejiang University, Hangzhou.

[6] Bear J, Porous Media Fluid Dynamics. Beijing: China Building Industry Press, 1983.

[7] Jin Longzhe, Li Jinping, Theory of Mine Dust Prevention and Control. Beijing: Science Press, 2010.

[8] Zhou Gang, Cheng Weimin, Chen Lianjun, Research and Practice of Key theory and Technical Process of Mine Dust Control, Beijing: Coal Industry Press, 2011.

[9] Gong Lihong, "Experimental Study on Factors Affecting Dust Removal Performance of Wet Vibration Fiber Grid," Building Energy Ventilation and Air Conditioning, vol. 23, no. 3, pp. 86-89, 2004.

[10] Liu Jun, Jin Shuqing, Xiang Xiaodong, "Gas-liquid separation mechanism and experimental study of wet vibrating wire dust collector," Journal of Wuhan University of Science and Technology, vol. 36, no. 4, pp. 299-302, 2013.

[11] Yao Xiaoqing, "Study on dust filtration performance of wet dense fiber grid," Qingdao Technological University, Qingdao.

[12] Zhao Jie, Wang Haiqiao, Chen Shiqiang, "Experimental study on the resistance characteristics of wet resonant grid," Journal of Heilongjiang University of Science and Technology, vol. 25, no. 1, pp. 82-86, 2015.

[13] Luo Zhenjiang, Zhao Jie, "Dust Removal Technology and Application of Xinnanfeng Well in Fankou Lead-Zinc Mine," Mining Engineering Research, vol. 30, no. 1, pp. 26-29, 2015.

[14] Xiang Xiaodong, Theory, Technology and Application of Soot Fiber Filtration. Beijing: Metallurgical Industry Press, 2007.

[15] Wang Liping, Chen Jianping, Air Pollution Control Engineering. Xuzhou: China University of Mining and Technology Press, 2012.

[16] Gong Lihong, "Theoretical and experimental research on wet vibrating fiber grating dust removal technology," Northeastern University, Shenyang.

[17] Cai Zengji, Long Tianqi, Fluid Mechanics Pumps and Fans. Beijing: China Building Industry Press, 1999.

[18] Lu Haizhu, "Application of Spray Dust Reduction in Fankou Lead-Zinc Mine," Industrial Safety and Environmental Protection, vol. 28, no. 9, pp. 35-36, 2002. 